\title{
Searching for New Leads To Treat Epilepsy: Target-Based Virtual Screening for the Discovery of Anticonvulsant Agents
}

Pablo H. Palestro, ${ }^{\dagger}$ Nicolas Enrique, ${ }^{\ddagger}$ Sofia Goicoechea, ${ }^{\dagger}$ Maria L. Villalba, ${ }^{\dagger}$ Laureano L. Sabatier, ${ }^{\dagger}$ Pedro Martin, ${ }^{\ddagger}$ Veronica Milesi, ${ }^{\ddagger}$ Luis E. Bruno Blanch, ${ }^{\dagger}$ and Luciana Gavernet ${ }^{*}{ }^{\dagger}$ (i)

${ }^{\dagger}$ Medicinal Chemistry, Department of Biological Sciences, Faculty of Exact Sciences, National University of La Plata, 47 and 115 , La Plata, Buenos Aires B1900BJW, Argentina

${ }^{\ddagger}$ Instituto de Estudios Inmunológicos y Fisiopatológicos (IIFP, CONICET-Universidad Nacional de la Plata), Fac. de Ciencias Exactas, Universidad Nacional de La Plata, La Plata, Buenos Aires B1900BJW, Argentina

Supporting Information

ABSTRACT: The purpose of this investigation is to contribute to the development of new anticonvulsant drugs to treat patients with refractory epilepsy. We applied a virtual screening protocol that involved the search into molecular databases of new compounds and known drugs to find small molecules that interact with the open conformation of the Nav1.2 pore. As the 3D structure of human Nav1.2 is not available, we first assembled 3D models of the target, in closed and open conformations. After the virtual screening, the resulting candidates were submitted to a second virtual filter,
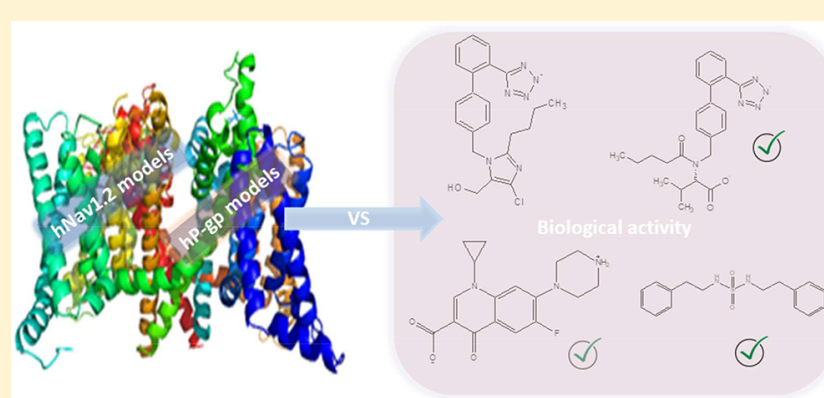
to find compounds with better chances of being effective for the treatment of P-glycoprotein (P-gp) mediated resistant epilepsy. Again, we built a model of the 3D structure of human P-gp, and we validated the docking methodology selected to propose the best candidates, which were experimentally tested on Nav1.2 channels by patch clamp techniques and in vivo by the maximal electroshock seizure (MES) test. Patch clamp studies allowed us to corroborate that our candidates, drugs used for the treatment of other pathologies like Ciprofloxacin, Losartan, and Valsartan, exhibit inhibitory effects on Nav1.2 channel activity. Additionally, a compound synthesized in our lab, $N, N^{\prime}-$ diphenethylsulfamide, interacts with the target and also triggers significant Na1.2 channel inhibitory action. Finally, in vivo studies confirmed the anticonvulsant action of Valsartan, Ciprofloxacin, and $N, N^{\prime}$-diphenethylsulfamide.

\section{INTRODUCTION}

Anticonvulsant drugs (ACD) are the mainstay of antiepileptic therapy. ${ }^{1}$ During the past decades, drug treatment of epilepsy has made significant progress by improving the number of new medications to control or prevent the seizures. However, two major problems remain unsolved in terms of clinical outcome: the development of drug resistant epilepsy and the occurrence of severe toxic effects caused by known ACD in responsive patients. ${ }^{1}$ These limitations faced in therapy have been the subject of extensive analysis. ${ }^{2,3}$ Experts in the field have concluded that more effective drugs can be designed if we incorporate in the early stages of the drug discovery process the actual knowledge of the molecular alterations that generate epileptic seizures and the new structural and functional information about the molecular targets of the candidates (e.g., Vigabatrin, Tiagabine, Perampanel). ${ }^{3}$ However, the drugs found by this rational approach represent an exception in the history of ACD, since screening campaigns on animal models of epilepsy have been almost the exclusive strategy for identifying the marketed compounds. Particularly, the maximal electroshock seizure (MES) test and the pentylenetetrazol seizure (PTZ) test are the biological assays most widely used. They are included into the NIH anticonvulsant drug development program (ADD program) to initially evaluate promising structures as anticonvulsants. ${ }^{4}$

As a general picture, the primary mechanisms of action of most ACD can be grouped into three main classes involving: (1) inhibition of voltage-activated ion channels (such as sodium and/or calcium), (2) increase of $\gamma$-aminobutyric acid (GABA) levels in neural GABAergic pathways through intervention in its synthesis, transport, release, and degradation, and (3) inhibition of glutamatergic excitatory synapses by a direct interaction of ACD with glutamate receptors or indirectly by precluding the glutamate liberation. ${ }^{2,5}$ However, other alternative mechanisms of action have been identified for novel structures. ${ }^{6,7}$ For example, synaptic modulation through vesicle protein SV2A is the main mechanism for Levetiracetam and Brivacetam, ${ }^{8}$ and the recently withdrawn drug Retigabine is known as a neuronal Kv7 (KCNQ) potassium channel modulator. ${ }^{6}$ Additionally, new candidates in clinical trials interact with new and different targets such as the cannabinoid system, the mammalian target of rapamycin (mTOR),

Received: December 15, 2017

Published: June 5, 2018 
acetylcholinesterase $(\mathrm{AChE})$, serotonin receptors $(5 \mathrm{HT})$, and others. $^{7}$

In this investigation, we focused on the identification of new ACD that act through the blockage of voltage-gated sodium channels (VGSCs). These ion channels are involved in the mechanism of action of many ACD with probed clinical efficacy (e.g., Phenytoin, Lamotrigine, Carbamazepine, Oxcarbazepine, Eslicarbazepine, Zonisamide, and Lacosamide). 3,9,10 In particular, we selected the VGSC isoform Nav1.2 as the molecular target of this investigation, since it is involved in epileptic disorders and it has confirmed interaction with the clinically relevant ACDs. ${ }^{3,11}$

From a structural point of view, eukaryote VGSCs are complex membrane proteins. They consist of a pore-forming $\alpha$-subunit in association with one or more $\beta$-subunits. ${ }^{12}$ Nine different $\alpha$-subunits (Nav1.1-Nav1.9) have been identified with four of them mainly expressed in the central nervous system (Nav1.1, Nav1.2, Nav1.3, and Nav1.6). ${ }^{13}$ The $\alpha$ subunit comprises four domains (DI-DIV) each containing six membrane-spanning segments (S1-S6). The four domains contribute to the channel's voltage sensor (transmembrane helices S1-S4) and to the ion conducting pore. ${ }^{12}$ The voltage sensor is responsible for voltage-mediated gating of the pore by detecting changes in the transmembrane voltage through positive amino acids located at every fourth position of the highly conserved S4 segment. The pore is formed by S5 and S6 helices, linked by P-loops (P1 and P2 helices). ${ }^{12}$

The searching of isoform selectivity to a specific target constitutes a usual approach in the drug design process, mainly focused on reducing adverse effects. In this investigation, we also considered functional selectivity, that is, the capacity of ACD to change their affinity depending on the conformational state of this ion channel target. ${ }^{10}$ The VGSCs can adopt at least three different conformational states depending on the voltage across the membrane (Figure 1). In neurons, at negative membrane potentials $(-80 \mathrm{mV}$ or less), most of them are in a resting state (closed conformation) and they activate in response to plasma membrane depolarization, allowing the conformational change from a closed to an open state that mediates an inward $\mathrm{Na}^{+}$current. During the depolarization phase of the action potential, the channels pass from the open

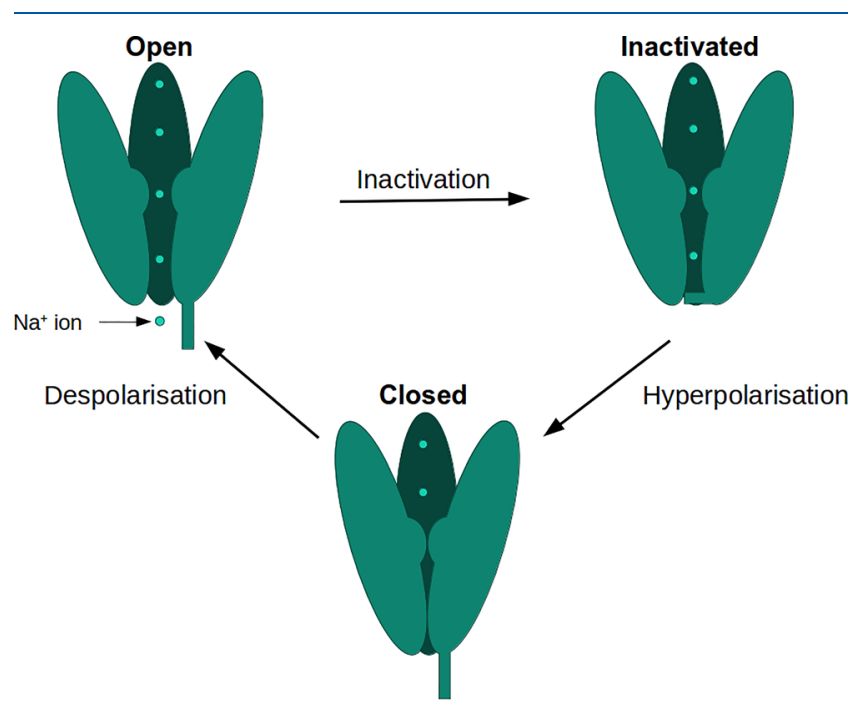

Figure 1. Three state model of VGSC. ${ }^{15}$ The conformations are dependent on the voltage across the membrane. state to a new nonconductive conformational state, the inactivated state. ${ }^{5}$ Some ACD show better affinity for the open and inactivated conformations of the VGSCs than for the closed one. ${ }^{5,9-11,14}$ This allows functional selectivity based on the fact that ACD act only when VGSCs have reached one of these states. ${ }^{10}$ As the epileptic seizures are characterized by the increase of the neuronal excitability, there are high probabilities to find the channels in the open and inactivated state. ${ }^{10,11}$

On the other hand, one of the major problems of current ACD therapy is the development of drug-resistant epilepsy. Several causes have been proposed to explain this phenomenon. ${ }^{16}$ Among them, the transporter hypothesis suggests that the expression and activity of efflux transporters, such as Pglycoprotein (P-gp), prevent the access of ACD into their molecular targets in a subset of patients. ${ }^{16,17}$

In this investigation, we applied a sequential virtual screening campaign to find new compounds that interact with the open conformation of the Nav1.2 pore but not with Pgp efflux transporter. As the 3D structure of human Nav1.2 is not available, initially, we constructed 3D models of the target, in closed and open conformations. After the virtual screening, the resulting candidates were submitted to a second virtual filter, in order to find compounds with better chances of being effective for the treatment of P-gp mediated resistant epilepsy. Again, no human 3D structure of P-gp is available, so we used several models of the macromolecule and we chose the docking protocols that best replicate the experimental data for the screening.

The sequential search was performed over databases that include FDA-approved compounds, nutraceuticals, and drugs in the experimental stage. To find anticonvulsant activities in compounds that belong to the universe of available drugs is a strategy known as drug repositioning, and it has several advantages over the traditional drug development process. ${ }^{18}$ For example, these compounds have already been tested in a significant number of toxicity assays, so the rate of failure due to adverse effects in the following steps of the drug discovery pipeline is lower than the one predicted for new compounds. ${ }^{18}$ Similarly, known drugs have successful and well documented pharmacokinetic studies, that reduce the time of the drug development process. ${ }^{19}$ We also included a homemade virtual database that comprises structures synthesized in our laboratory. Previous investigations from our research group and others pointed to sulfamide as an attractive chemical entity with anticonvulsant properties. ${ }^{20-22}$ As a consequence of our investigations, we found sulfamide derivatives that outperform structurally related anticonvulsant drugs in terms of potency against the MES test. ${ }^{16-18}$ There is not a direct correlation between the activity in the MES test and the mechanism of action of the ACD, but traditional sodium channel blockers (such as Phenytoin, Lamotrigine, and Carbamazepine) are active in this assay. ${ }^{23,24}$ For that reason, we included in the virtual screening the sulfamide derivatives that were active in this acute model.

Finally, four candidates were evaluated on sodium channel activity applying the patch clamp technique on HEK293 cells expressing the Nav1.2 channel isoform and in vivo test through the traditional MES assay of convulsions in mice. All of them showed an inhibitory effect on the Nav1.2 channels, and three of them also demonstrated anticonvulsant activity in vivo. 
A)
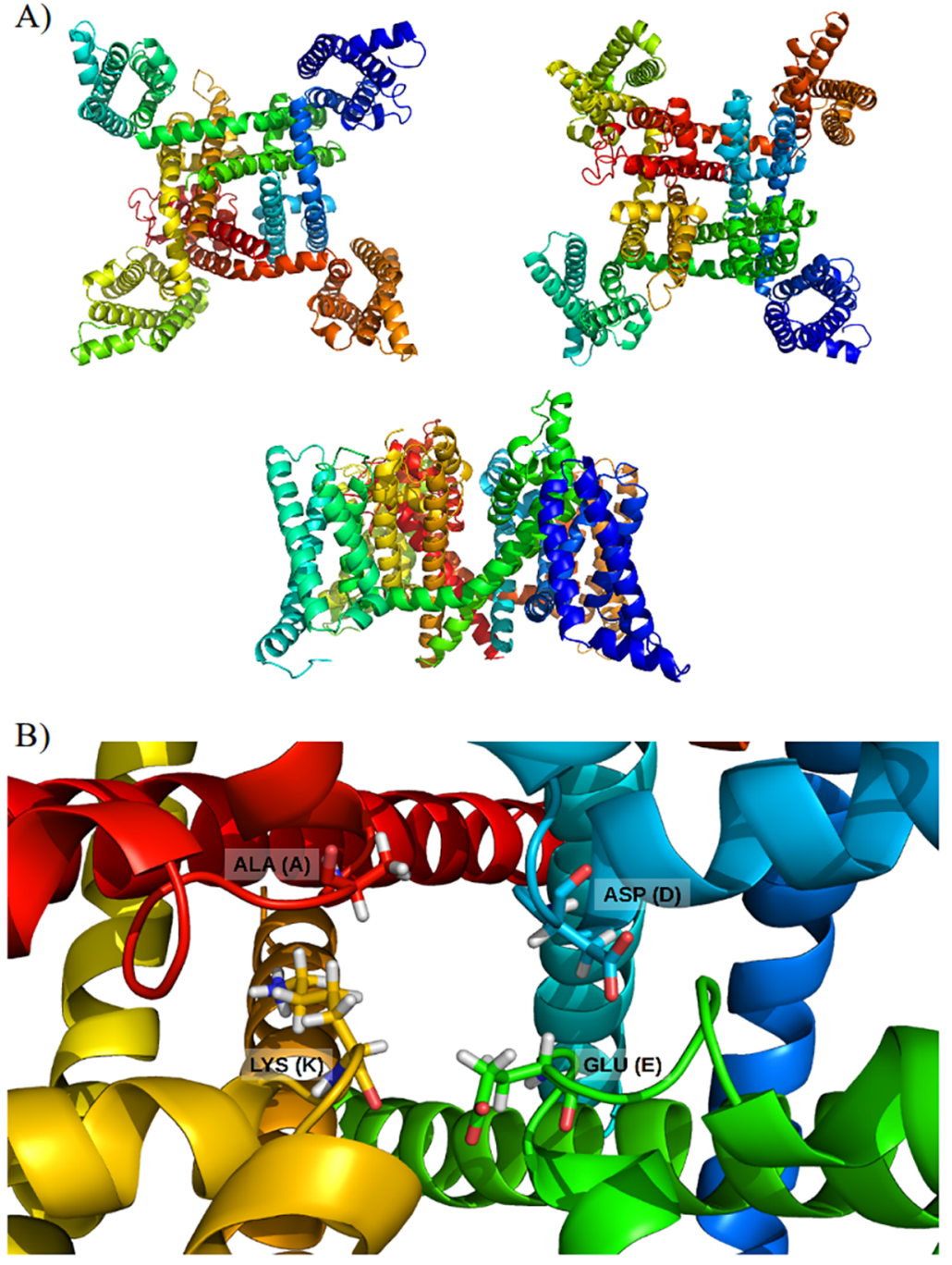

Figure 2. (A) Final model of the sodium channel (isoform Nav1.2) in a closed conformation (top and side view). (B) DEKA ring.

\section{RESULTS}

2.1. Construction of Human Nav1.2 Models. Initially, we modeled the 3D structure of the Nav1.2 isoform in the closed and open conformations. We constructed the $\alpha$-subunit of the channel, which is functional on its own and contains the region of interactions with ACD (which is located in the conducting pore of the macrostructure). ${ }^{13-24}$ The closed pore conformation was built by means of the GPCR-I-TASSER server, which is based on the protein threading server ITASSER but incorporates restrictions associated with transmembrane proteins. ${ }^{25}$ The four domains were modeled separately, and the best solution of each one was selected on the basis of the metric provided by the server for estimating the quality of the predictions. The values of C-score of the best models varied between -0.59 and -2.47 depending on the domain, and the TM-scores were higher than 0.43 (the Cscore is typically in the range of -5 to 2 and a TM-score lower than 0.17 means random similarity). More details are given in Table S1. Once the domains were constructed, the final heterotetrameric structure was assembled by superposition with the crystal structure of bacterium Arcobacter butzleri $\left(\mathrm{NavAb}\right.$, protein data bank (PDB) ${ }^{26}$ entry: $\left.3 \mathrm{RVY}^{27}\right)$. This template emerged in the threading procedure, and it showed the highest percentage of sequence coverage in the alignment process with NCBI-BLAST server. Finally the whole $\alpha$-subunit of the channel was submitted to energy minimization with the SANDER module of AMBER11 software ${ }^{28}$ (details are given in the Supporting Information). Figure 2A shows the overall architecture of the final model. Ramachandran plots confirmed the high quality of the model, with $97.6 \%$ of residues placed in the allowed regions and few amino acids (2.4\%) with unfavorable positions (Figure S1). Another important structural aspect reproduced in the model is the presence of the "DEKA" motif. Eukaryotic sodium channels host a highly conserved selectivity filter of ions, located at the P-loops that connect the S5 and S6 helices of the four domains. ${ }^{29}$ This filter is composed of four amino acid side chains of Asp (D), Glu (E), Lys (K), and Ala (A) residues, placed in domains I to IV, respectively. Figure $2 \mathrm{~B}$ shows the location of the DEKA ring in the model. It is important to note that none of the outlier residues identified in the Ramachandran plot are part of the defined active site for the docking simulations or the DEKA motif. Additionally, our model was correctly classified as a membrane protein by QMEANbrane software, ${ }^{30}$ which also identified the pore as the transmembrane region of the model (Figure S2). 


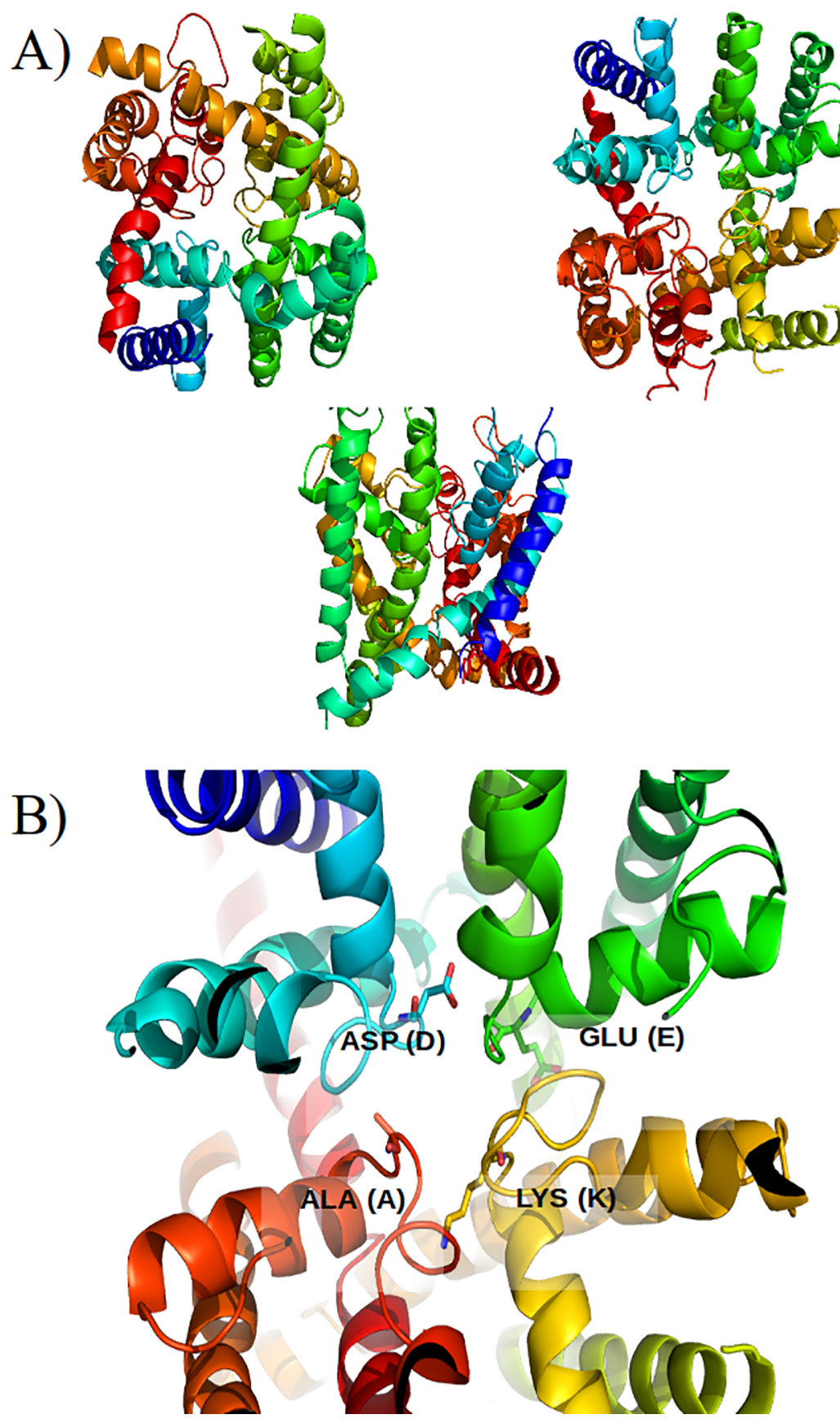

Figure 3. (A) Final model of the sodium channel (isoform Nav1.2) in an open conformation (top and side view). (B) DEKA ring.

As highlighted in the previous paragraph, the architecture of the sodium channel in bacterium was used for assembling the domains of human Nav1.2 channel. The selection of this pattern is supported by the parameters found in the alignment process, but it shows a closed conformation. To predict the 3D architecture of the target in the open pore conformation, we modified the template, following the strategy applied by Tomašic et al. for the construction of the open pore conformation model of the human Nav1.4 isoform. ${ }^{31}$ The authors analyzed the structural data available for the nonselective NaK cation channel of Bacillus cereus. Interestingly, there are experimental structures in the open and closed pore conformation of the $\mathrm{NaK}$ channel for this bacterium (PDB entries: $3 \mathrm{E} 86$ and $2 \mathrm{AHY}$, respectively), that allow us to suggest where the main structural differences are between the two states. The superposition of the two experimental structures of $\mathrm{NaK}$ channels showed that these two conformations diverge mostly in the inner region of the pore, below the selectivity filter (Figure S3). The same observation can be accomplished with the comparison between the open conformation of the $\mathrm{NaK}$ channel (PDB entry: 3E86) and the NavAb template for the closed state (PDB entry: RVY). Particularly, the major changes can be observed in the coordinates of the S6 helices that comprise the pore of the channel (Figure S3). Therefore, we imitated the "opened pore template" constructed by Tomašic et al. for Nav1.4 models by replacing the coordinates of the S6 segment of NavAb by the ones obtained for the open pore conformation of the $\mathrm{NaK}$ channel. ${ }^{31}$ It is important to mention that the structural changes in the S6 segments are accompanied by modifications in the position of the S4 
segments (voltage sensor). The change of position of S4 helices cannot be modeled in the open pore conformation since they are absent in $\mathrm{NaK}$ template, so we deleted them for the construction of the model, to avoid the superimposition of S4 with the S6 segment. ${ }^{32}$ In other words, the open model of the human Nav1.2 channel built for this investigation included the segments S5 and S6 of the four domains of the channel. The S5 and S6 portions constitute the pore of the macrostructure and contain the active site of ACD, so they are valuable for the virtual screening campaign proposed in this investigation. Initially, the sequences of S5 and S6 segments of each domain were aligned by means of homology methods with MODELLER software. ${ }^{33-36}$ The solutions with the highest discrete optimized protein energy (DOPE) score were selected for each domain (values between 0.9 and 1.7, Table S1). Then, the pore was assembled with superposition of the domains with the open pore template. Finally, the model was submitted to energy minimization with the SANDER module of AMBER 11. ${ }^{28}$ Details are given in the Methods. The Ramachandran plots confirmed the high quality of the final model, with $91.9 \%$ of amino acids located in the allowed regions and few amino acids (8.1\%) with unfavorable positions (Figure S4). Again, none of the residues that comprise the active site or the selectivity filters are outliers in the Ramachandran plot, and QMEANbrane software correctly classified the model as a membrane protein with the pore into the transmembrane region (Figure S5). Figure 3 shows the final architecture of the open pore obtained for the human Nav1.2 model and the position of the DEKA ring, which was also well reproduced in this conformation.

2.2. Validation of the Docking Protocol for Nav1.2 Virtual Screening. With the models of Nav1.2 available, the next step was the selection of the docking conditions for virtual screening. We analyzed the ability of the software to discriminate known binders from nonbinders of Nav1.2 through the docking score. To this end, we carefully compiled a test set of 183 active compounds and 323 inactive structures mainly from the CHEMBL database ${ }^{37}$ (see Methods). Autodock Vina (flexible mode) ${ }^{38}$ was used for molecular docking in both closed and open conformation of the target. The active site was defined on the basis of the experimental data. ${ }^{39}$ The information available pointed to the residues numbered from Phe1764 to Tyr1771 as important for the interactions of the channel with known ACD. ${ }^{24,39}$

Figure 4 shows the receiver operating characteristic (ROC) curves for the best model of each conformation. The ROC curves plot the sensitivity of the model (true positive rate) as a function of the false positive rate $(1-$ specificity) at various threshold settings. ${ }^{40}$ Accordingly, a perfect classification of compounds would be represented in the graph by a line that starts from the origin, reaches vertically the upper left corner, and then goes to the upper right corner. The area under the curve (AUC) will be equal to one in this ideal performance while an AUC of 0.5 will represent a random selection of active and inactive compounds. The AUC values for our best docking models were 0.916 for the open conformation and 0.878 for the closed one, which demonstrated the capacity of the protocols to truly discern between active and inactive compounds. The lower value of AUC achieved for the closed conformation was expected if we consider that ACD have low affinities to the target in this state. The poorer interactions might have influence in the correct classification of known binders through the docking score. For that reason, we selected

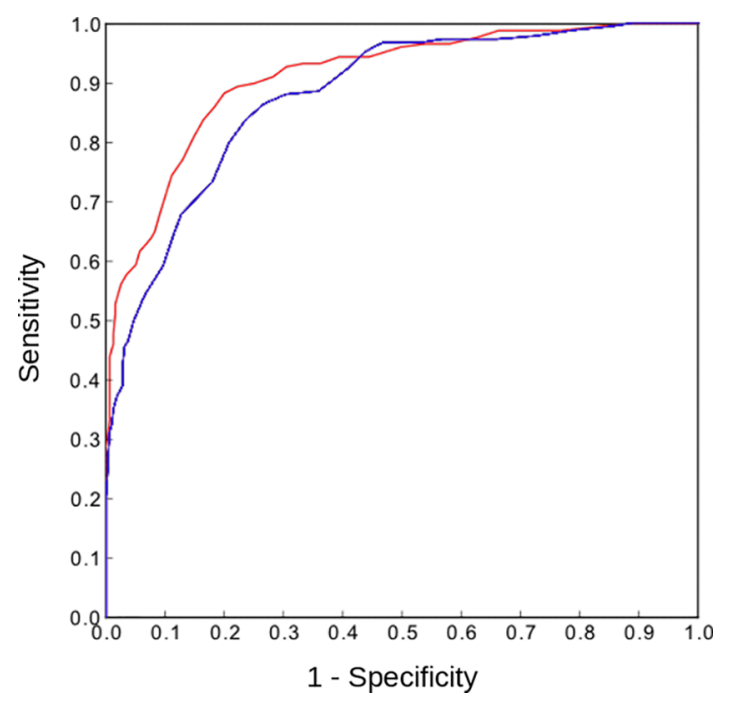

Figure 4. ROC-type curves obtained from docking simulations into the active site of the open (red) and closed (blue) models of Nav1.2.

the best docking conditions achieved for the simulation in the open structure, which is also more representative of the active conformation of the target.

2.3. Nav1.2 Virtual Screening. The docking protocol detailed before was used for the virtual screening of 9133 structures of FDA-approved compounds, nutraceuticals, and drugs in the experimental stage (available from DrugBank4. $0^{41}$ and ZINC-FDA databases, ${ }^{4}$ as detailed in Methods). A docking score of $-9.0 \mathrm{kcal} / \mathrm{mol}$ was defined as the threshold value to differentiate active from inactive compounds in the virtual screening of the database. This value has an associated specificity value of $93 \%$ and a sensitivity of $63 \%$ in the test set, according to the ROC curve. We decided to prioritize specificity over sensitivity to avoid the selection and purchase of false positives for the biological assays (at expenses of losing true positives during the search, due to the lower sensitivity rate). Additionally, 28 sulfamide derivatives synthesized in our laboratory were submitted for the virtual screening. ${ }^{20-22} \mathrm{We}$ set the docking threshold value to $-8.1 \mathrm{kcal} / \mathrm{mol}$ for this inhouse database, which is associated with a good balance between specificity and sensitivity for the test set $(80 \%$ and $88 \%$, respectively). We changed the cutoff value because in these conditions there are high chances to get true positive candidates ( $88 \%$ sensitivity), and we are particularly interested in these structures due to previous findings about their anticonvulsant activity in vivo (even at the risk of testing false positive compounds). ${ }^{20-22}$

Finally, the model classified as Nav1.2 blockers 1181 compounds from the ZINC database, 1596 structures from DrugBank, and 3 sulfamide derivatives from our lab. The selected structures were submitted to the second step in the virtual screening to avoid P-gp substrates.

2.4. Human P-gp Models and Validation of the Docking Protocol for Virtual Screening. P-glycoprotein is an important efflux transporter that belongs to the ATPbinding cassette $(\mathrm{ABC})$ family, and it is expressed in many barriers and excretory tissues. As other ABC transporters, P-gp has the important function of preventing the entrance of exogenous compounds into susceptible organs and cells. ${ }^{17} \mathrm{On}$ the other hand, P-gp may negatively affect the pharmacokinetic profile of new candidates by limiting the bioavailability of the 
drugs in the brain target thanks to its overexpression or activation. $^{17}$

In a preceding investigation, we constructed a P-gp human model and defined a validated docking protocol for virtual screening of P-gp substrates. ${ }^{43}$ Afterward, Szewczyk et al. deposited new experimental structures of mouse P-gp into the $\mathrm{PDB},{ }^{44}$ providing more information about the architecture of the active site and the binding mode of certain ligands.

In this investigation, we used our best docking protocol (Autodock Vina, flexible docking) ${ }^{43}$ to simulate the interaction of a new test set into the crystal structures of Szewczyk and coworkers (PDB codes 4Q9I, 4Q9J, 4Q9K, and 4Q9L). ${ }^{44}$ Interestingly, the authors identified two different locations and orientations of the ligands in the substrate-binding pocket of 4Q9I, 4Q9J, and 4Q9L. These findings are consistent with previous investigations that pointed to several binding sites for substrates and inhibitors of the glycoprotein. ${ }^{45,46}$ The two binding sites proposed in each crystal structure were considered separately as the docking region, and two different docking runs were performed for these macromolecules. The docking protocol was run over an extended test set of $1206 \mathrm{P}$ gp binders and nonbinders (23 times bigger than the original $^{43}$ ) for the comparison of the results with different structures of the antitarget. The set was taken from the database of 1275 binders and nonbinders provided by Broccatelli et al., ${ }^{47}$ which was manually curated. More information is given in the Methods.

Table 1 shows the results of the AUC calculated for the docking of the extended test set. Interestingly, the docking

Table 1. Values of AUC Achieved from the Docking Simulations of the Extended Test Set (1206 Compounds)

\begin{tabular}{llc}
\multicolumn{1}{c}{ target $^{a}$} & \multicolumn{1}{c}{ flexible residues $^{b}$} & AUC \\
4Q9I & Phe332_Tyr949_Phe979_Phe728 & 0.81 \\
4Q9I & Trp228_Ala225_Leu221_Phe339_Phe332 & 0.79 \\
4Q9J & Val978_Met982_Phe332_Met68_Phe71 & 0.76 \\
4Q9J & Trp228_Ala225_Leu221_Phe339 & 0.78 \\
4Q9K & Leu335_Phe332_Met982_Val978_Phe979_Ser725 & 0.81 \\
4Q9L & Gln721_Ser725_Phe979_Phe339 & 0.79 \\
4Q9L & Met945_Phe332_Phe339_Met982 & 0.80 \\
hP-gp model & Tyr-307, Tyr-953, Phe-343, Phe-978 & 0.92
\end{tabular}

${ }^{a} \mathrm{PDB}$ code of the macroestructures employed in the simulations. ${ }^{b}$ Amino acids of the target considered as flexible in the docking simulation.

protocol considering the P-gp human model kept its capacity to discriminate binders from nonbinders of the test set used in this investigation (1206 structures, AUC value of 0.92), which is considerably more challenging than the original set compiled previously (52 compounds, AUC 0.83). ${ }^{43}$ Additionally, the docking simulation based on the human model showed better performance than the calculations with the crystal structures of mouse glycoprotein, so it was selected for virtual screening.

2.5. P-gp Virtual Screening. The structures that survived the first Nav1.2 virtual screening were submitted to the P-gp filter, in order to eliminate compounds with high affinity to this protein. P-gp acts in the screening as an antitarget, so the best candidates should have a docking score higher than the threshold number. For the compounds recovered from ZINC and Drugbank databases, this value was set as $-8.5 \mathrm{kcal} / \mathrm{mol}$, which is able to predict $89 \%$ of the known binders (sensitivity value of 0.89 ) and the $55 \%$ of nonbinders (specificity value of $0.55)$.

As the numbers suggest, we prioritized sensitivity over specificity to identify correctly the positive ligands and consequently discard them after the screening. About 190 structures from ZINC and Drugbank were classified as Nav1.2 blockers and P-gp nonbinders. A careful analysis of the available literature showed that two of the candidates, Ropivacaine and Cibenzoline, have already been tested as sodium channel blockers. ${ }^{48-50}$ These findings provided us confidence in the capacity of the screening method to find active structures. Finally, three candidates were available for biological assays: Losartan and Valsaltan, two drugs used to treat hypertension by inhibiting angiotensin II receptor, and Ciprofloxacin, a fluoroquinolone-based antibiotic drug (Figure 5).

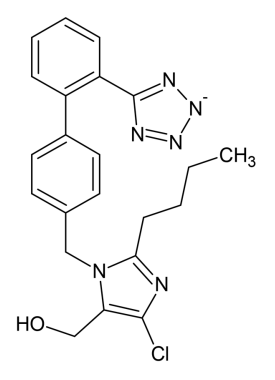

Losartan

Nav1.2: -9.7; P-gp: -8.5

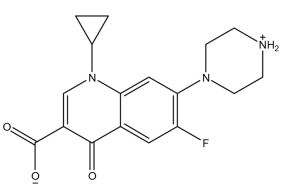

Ciprofloxacin

Nav1.2: -9.1; P-gp: -8.2

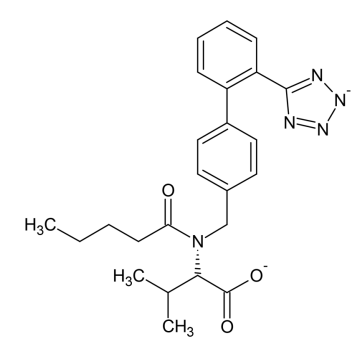

Valsartan

Nav1.2: -9.8; P-gp: -8.4

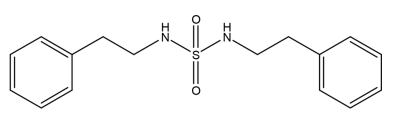

N, N'-diphenethylsulfamide

Nav1.2: -9.5; P-gp: -8.8
Figure 5. Chemical structures of the candidates and their docking scores for Nav1.2 and P-gp screening.

Regarding sulfamide derivatives, a more "balanced" restriction was applied for the threshold value, which was set as $-9.0 \mathrm{kcal} / \mathrm{mol}$ (sensitivity of $73 \%$ and specificity of $80 \%$ for the test set). Three candidates were classified as nonsubstrates of P-gp. Among them, $N, N^{\prime}$-diphenethylsulfamide showed the best score in the docking simulation with Nav1.2, so this compound was selected for biological evaluations (Figure 5).

2.6. Biological Assays. 2.6.1. Electrophysiological Assays. The patch clamp technique was used to measure the effect of the candidates on sodium channel activity. The ability of each candidate to change the sodium current mediated by hNav1.2 channels stably expressed in HEK293 cells was tested in the whole-cell configuration by applying a $15 \mathrm{~ms}$ voltage step from a holding potential of -70 to $-20 \mathrm{mV}$. In the same experimental conditions, we also tested the effect of $100 \mu \mathrm{M}$ Phenytoin, in order to compare the inhibitory potency of the candidates with this classic AED. The results are shown in Figure 6. All candidates produced a significant inhibitory effect on hNav1.2 currents. $N, N^{\prime}$-Diphenethylsulfamide was the most 
A

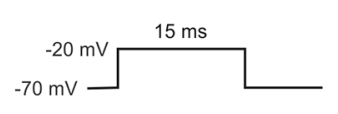

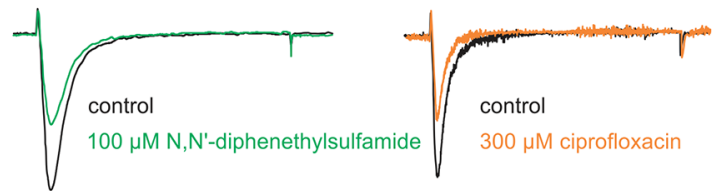
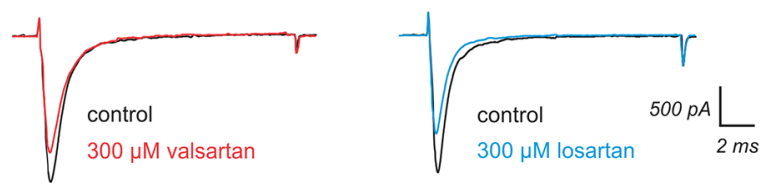

B

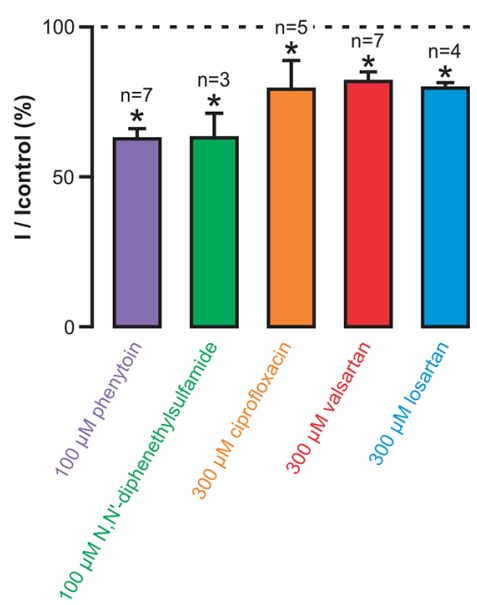

Figure 6. Effect of the candidates and Phenytoin on whole-cell $\mathrm{Na}^{+}$currents mediated by hNav1.2 channels. Color code as follows: Phenytoin in violet, $N, N^{\prime}$-diphenethylsulfamide in green, Ciprofloxacin in yellow, Valsartan in red, Losartan in blue, and control solutions without compound in black. (A) Results obtained from a typical whole-cell voltage-clamp protocol (holding potential of $-70 \mathrm{mV}$ and test potential of $-20 \mathrm{mV}$ ) applied under control conditions or in the presence of the indicated compounds. (B) Mean values \pm ESM of the relative current blockage of $\mathrm{Na}^{+}$currents by each drug. Statistically significant differences showing a $p<0.05$ were marked with an asterisk (paired $t$ test).

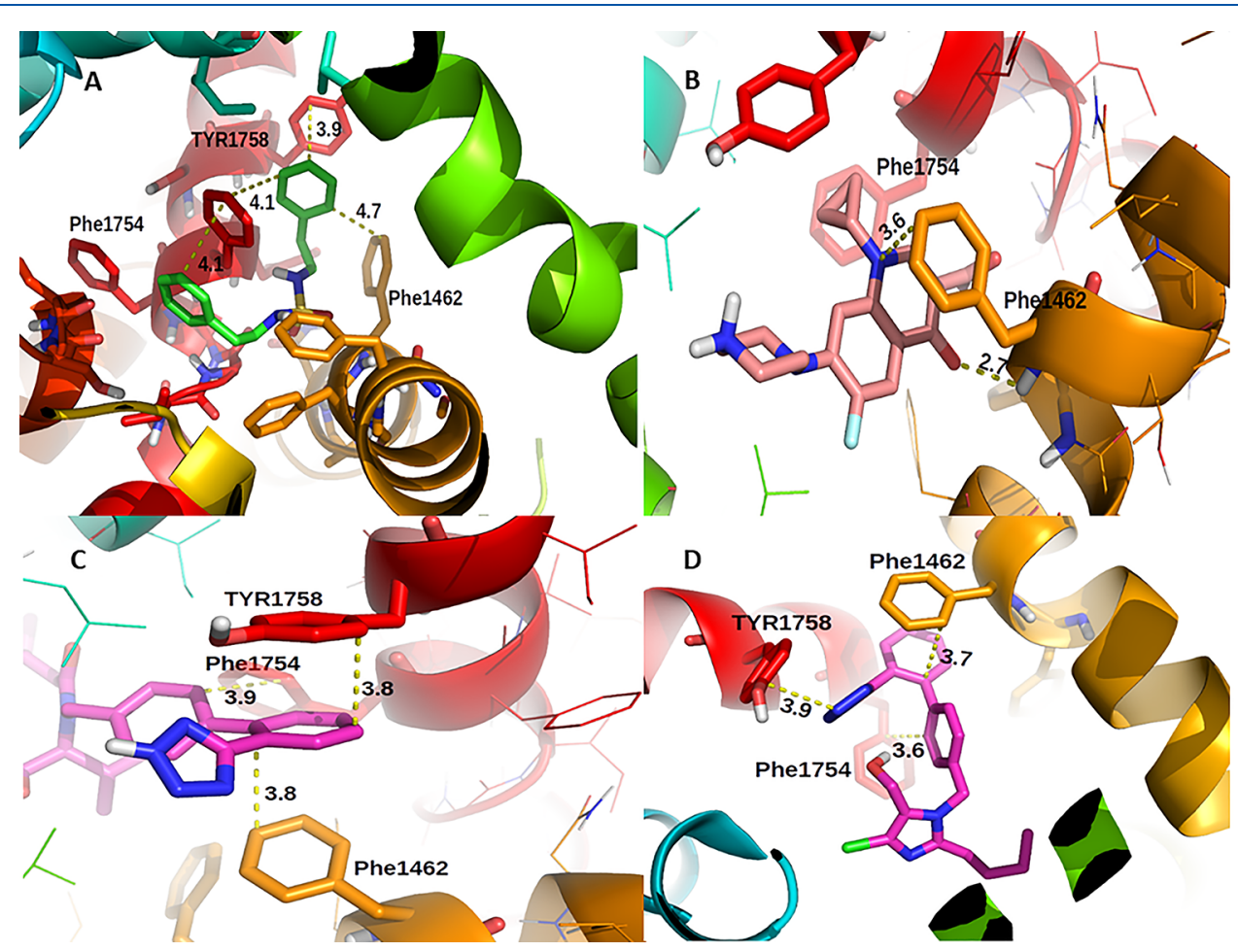

Figure 7. Interactions predicted by docking simulations between the candidates and Nav1.2. (A) N,N'-Diphenethylsulfamide (carbon atoms in green, nitrogen atoms in blue). (B) Ciprofloxacin (carbon atoms in pink, nitrogen atoms in blue). (C) Valsartan (carbon atoms in purple, nitrogen atoms in blue). (D) Losartan (carbon atoms in purple, nitrogen atoms in blue, chlorine atom in green). Hydrogen atoms attached to carbon atoms were omitted for simplicity.

potent candidate showing a relative $\mathrm{Na}^{+}$current reduction similar to that obtained with Phenytoin, both tested at $100 \mu \mathrm{M}$ concentration $(36.9 \% \pm 8.2 \%, n=3, p<0.05$ and $37.4 \% \pm$ $3.4 \%, n=6, p<0.05$, \% of current inhibition with $N, N^{\prime}-$ diphenethylsulfamide and Phenytoin, respectively). Additionally, Ciprofloxacin, Losartan, and Valsartan were also able to inhibit the hNav1.2 currents at $300 \mu \mathrm{M}$ concentration $(20.9 \%$ $\pm 4.8 \%, n=5 ; 20.3 \% \pm 1.9 \%, n=4 ; 18.2 \% \pm 3.2 \%, n=7$, respectively).
Figure 7 shows the results of the docking simulation of the candidates into the active site of Nav1.2 (open conformation). According to our predictions, lipophilic and $\pi$-stacking interactions between the $N, N^{\prime}$-diphenethylsulfamide and the active site play an important role in the binding event. Particularly, aromatic side chains of Phe1462, Phe1754, and Tyr1758 interact with the phenyl substituents of the candidate. Valsartan, Losartan, and Ciprofloxacin showed a similar type of interaction to a lesser extent. Additionally, hydrogen bonding 
interactions were detected between Ciprofloxacin and the nitrogen atom of the backbone of Phe1462.

2.6.2. In Vivo Assays. The biological evaluation of the candidates in mice was performed following the standard procedures proposed by the NIH anticonvulsant drug development $(\mathrm{ADD})$ program, via the anticonvulsant screening project (ASP). ${ }^{4}$ We tested the compounds against the MES test because there is evidence about the inhibitory effects on the convulsions caused in this assay by the ACD that acts as sodium channels blockers. ${ }^{23}$ We also measured primary toxicity of the drugs (sedation and ataxia) with the standardized RotoRod test, which is also included in the primary phase of the ADD program. ${ }^{4}$ The compounds were administrated to animals (mice) intraperitoneally at the lower doses of the program $(30,100 \mathrm{mg} / \mathrm{kg})$, and all the assays were performed at 0.5 and $4 \mathrm{~h}$. Details are given in the Methods. The results obtained from the evaluation are shown in Table 2, which included the data already reported for $N, N^{\prime}$-diphenethylsulfamide. $^{21}$

Table 2. Anticonvulsant and Neurotoxic Effects of the Candidates in Mice

\begin{tabular}{|c|c|c|c|c|c|}
\hline \multirow[b]{2}{*}{ compound } & \multirow{2}{*}{$\begin{array}{c}\text { dose } \\
(\mathrm{mg} / \mathrm{kg})\end{array}$} & \multicolumn{2}{|c|}{ MES test ${ }^{a}$} & \multicolumn{2}{|c|}{$\begin{array}{c}\text { RotoRod } \\
\text { test }^{b}\end{array}$} \\
\hline & & $0.5 \mathrm{~h}$ & $4 \mathrm{~h}$ & $0.5 \mathrm{~h}$ & $4 \mathrm{~h}$ \\
\hline \multirow[t]{2}{*}{$N, N^{\prime}$-diphenethylsulfamide ${ }^{17}$} & 30 & $0 / 3$ & $0 / 3$ & $0 / 3$ & $0 / 3$ \\
\hline & 100 & $3 / 3$ & $0 / 3$ & $0 / 3$ & $0 / 3$ \\
\hline \multirow[t]{2}{*}{ Losartan } & 30 & $0 / 2$ & $0 / 2$ & $0 / 2$ & $0 / 2$ \\
\hline & 100 & $0 / 2$ & $0 / 2$ & $0 / 2$ & $0 / 2$ \\
\hline \multirow[t]{2}{*}{ Valsartan } & 30 & $0 / 2$ & $0 / 2$ & $0 / 2$ & $0 / 2$ \\
\hline & 100 & $1 / 2$ & $1 / 2$ & $0 / 2$ & $0 / 2$ \\
\hline \multirow[t]{2}{*}{ Ciprofloxacin } & 30 & $0 / 2$ & $2 / 4$ & $1 / 2$ & $0 / 4$ \\
\hline & 100 & $0 / 2$ & $1 / 4$ & $0 / 2$ & $0 / 2$ \\
\hline
\end{tabular}

${ }^{a}$ Number of protected animals relative to the total number of mice tested in Maximal Electroshock Seizure at each time and concentration. ${ }^{b}$ Number of animals with sedative effects relative to the total number of mice tested at each time and concentration.

No anticonvulsant activity was observed for Losartan at the tested doses. Valsartan showed anticonvulsant action with 50\% of animals protected at both 0.5 and $4 \mathrm{~h}$ after administration (dose: $100 \mathrm{mg} / \mathrm{kg}$ ). Regarding Ciprofloxacin, it showed a remarkable positive response at both tested concentrations (4 $\mathrm{h}$ after administration), with $50 \%$ of protection at the lower dose. According to these results, Valsartan, Ciprofloxacin, and $N, N^{\prime}$-diphenethylsulfamide are the most promising structures for future analysis. It is important to mention that Ciprofloxacin evidenced sedative effects in one mouse (Rotorod test), so more experiments will be performed at lower doses to see if the anticonvulsant action prevails without these adverse effects.

\section{CONCLUSIONS}

The universe of anticonvulsant drugs collects a set of diverse structures that exert their action through the interaction with many biological targets. The actual knowledge of these targets at the molecular level (and their alterations that generate seizures) provides the opportunity to find new compounds via target-based drug discovery strategies. In this investigation, we constructed the structure of human Nav1.2 and validated the docking protocol employed for the selection of candidates in virtual screening. Additionally, we integrated a revalidated P-gp filter to avoid the interactions of the compounds with this efflux transporter. Electrophysiological assays showed that all the selected structures were able to inhibit $\mathrm{Na}^{+}$currents, suggesting the interaction of these compounds with the Nav1.2 channel. Moreover, in vivo assays showed that only Losartan failed to protect animals against the MES test.

The use of the patch-clamp technique applied to a heterologous system is the gold standard to validate the predictions of the human Nav1.2 structural model since the compounds are tested on the current carried by this specific channel isoform. ${ }^{14}$ However, the positive results obtained by this approach do not guarantee a direct correlation with in vivo effects. Differences between in vitro and in vivo assays can be explained by several factors that depend on: (i) the target, (ii) the selectivity of each compound, and/or (iii) its pharmacokinetics. (i) The pharmacological sensitivity of Nav channels can be modified by post-transcriptional modifications such as alternative splicing, channel protein phosphorylation, and/or glycosylation. The expression of auxiliary $\beta$-subunits and colocalization with other membrane proteins in native cells can also modify the activity of the channel blockers. ${ }^{51}$ (ii) Some AEDs are able to exert their antiepileptic effect by acting in multiple targets. ${ }^{52}$ Most of them are nonselective for neuronal Nav channels isoforms, and they are able to modify other ions channels (such as $\mathrm{K}^{+}$and $\mathrm{Ca}^{2+}$ channels), inotropic receptors (like GABA-A and NMDA), and enzymes (GABA transaminase and glutamic acid decarboxylase). ${ }^{3,7}$ (iii) In some cases, the drug candidates are unable to reach a significant concentration on the target environment when they are tested in vivo. The drug metabolisms and their ability to cross the blood-brain barrier are critical to obtain significant concentrations in the central nervous system, but these factors do not influence its in vitro activity. ${ }^{53}$ Some of these factors could explain the observed protection of Valsartan and Ciprofloxacin in vivo, in spite of their low inhibitory potency in the heterologously expressed Nav1.2 channel.

On the other hand, we observed that $N, N^{\prime}$-diphenethylsulfamide and Phenytoin exhibited similar effects on the Nav1.2 currents. However, Phenytoin is 10 -fold more potent in the MES test in mice than our candidate $\left(\mathrm{ED}_{50}: 34.5\right.$ and 263 $\mu \mathrm{mol} / \mathrm{kg}$ for Phenytoin and $N, N^{\prime}$-diphenethylsulfamide, respectively). ${ }^{21,54}$ This fact could be explained by differences in the selectivity and the pharmacokinetics profile of both compounds. ${ }^{52}$ Our results provide us the opportunity for further structural optimization based on the interactions with Nav1.2 proposed by molecular modeling.

\section{METHODS}

4.1. Molecular Modeling. 4.1.1. Construction of Nav1.2. The closed pore conformation was built with the GPCR-ITASSER server. ${ }^{25}$ The input sequence was taken from UNIPROT $^{55}$ (code Q99250), and the four domains were modeled individually. To this end, we defined domains I, II, III, and IV as the amino acids numbered 111-456, 741-1013, 1190-1504, and 1513-1611, respectively. The selection was based on the search of conserved domains in the NCBIBLAST server. The final macrostructure was assembled by superimposition with a NavAb template (PDB entry: 3RVY). The open pore conformation was assembled as explained in the Results.

Both closed and open geometries were minimized (1000 cycles for the water molecules followed by 2500 cycles for the 
entire systems) using the ff03.r1 version of the AMBER force field. ${ }^{28}$

4.1.2. Docking Protocol for Nav1.2. To select the docking software and conditions, we first constructed the active subset with 180 compounds that interact with Nav1.2 ( IC $_{50} \leq 100$ $\mu \mathrm{M})$ reported in the CHEMBL database. ${ }^{56}$ Additionally, we included some $\mathrm{ACD}$ with proved interaction with the target: Phenytoin, Carbamazepine, and Lamotrigine. ${ }^{23}$ Unfortunately, we have poor information about inactive compounds. We found that structures like Deprenyl, Gabapentin, Mecamylamine, Moclobemide, Nialamide, Procainamide, Tiapride, and Topiramate do not have affinity with Nav1.2. ${ }^{57}$ In order to increase the number of inactive compounds (and to better simulate the conditions expected in virtual screening of big databases), we performed a similarity search into the ZINC database ${ }^{42}$ with the inactive compounds as templates. We assumed that structures with similar characteristics will also be inactive against the target. The similarity threshold was set from $70 \%$ (Nialamide) to $99 \%$ (Mecamylamine), depending on the number of similar compounds recovered from each template. The final test set has 506 structures, 183 defined as active and 323, as inactive.

Autodock Vina (flexible mode) was used for the molecular docking of the test set in both closed and open conformation of Nav1.2. The docking active site was set according to experimental data, which pointed to the pore region as the place for interaction of ACD and local anesthetics. ${ }^{24,39}$ This area covers the amino acids numbered from Phe1764 to Tyr1771. We defined the grid size of $16 \times 19 \times 25 \mathrm{~A}^{3}$ for the closed conformation and $20 \times 20 \times 20 \mathrm{~A}^{3}$ for the open conformation. Flexible residues were also considered during the simulation: Gln383, Ser413, Phe1764, and Tyr1771 side chains were allowed to move in the closed conformation whereas Phe1462, Ser1463, Phe1756, and Tyr1760 were allowed to rotate in the open state.

4.1.3. Docking Protocol for P-gp. In previous investigations, we constructed a human P-gp model mainly based on the structure encoded as $3 \mathrm{G} 61$ in PDB, which showed the highest sequence coverage (92\%) and percent of identity (82\%) among other templates. ${ }^{43}$ Then, we docked into this macrostructure a test set of 26 binders and 26 nonbinders of $\mathrm{P}$ gp which was built on the basis of a careful analysis of the experimental data available. ${ }^{58-61}$ The comparison of several types of docking software and conditions allowed us to conclude that Autodock Vina flexible docking is the best choice among the tested options, ${ }^{43}$ and we used this model in conjunction with ligand-based virtual screening to successfully find new anticonvulsant compounds. ${ }^{62,63}$ In this investigation, the compounds included in the test set for the validation of the docking simulations were taken from the database of Broccatelli et al., ${ }^{47}$ which comprise 666 binders and 609 nonbinders of the protein. After the analysis of the literature, we were able to identify reliable data for 1206 of these compounds, so they were used to define our extended test set. In addition to our previously reported model of the $3 \mathrm{D}$ structure of human $\mathrm{P}$-gp, ${ }^{43}$ we utilized the experimental structures of mouse P-gp encoded as 4Q9I, 4Q9J, 4Q9K, and 4Q9L in the PDB for the docking of the set. In all cases, the crystallographic ligands were stripped, and hydrogen atoms were added using the leap module of AMBER11. ${ }^{28}$ The "docking active site" for each macrostructure was defined through a grid centered on the crystallographic ligand. As mentioned before in the text, most of the complex shows two ligands simultaneously crystallized into the protein (PDB codes 4Q9I, 4Q9J, and 4Q9L). Thus, we considered both possibilities as the docking active site. We used a $20 \times 20 \times 20$ $\mathrm{A}^{3}$ grid size and performed 100 docking runs for each compound using the default parameters for the rest of the variables. We computed rigid and flexible docking (data not shown), and the best solutions were obtained by the inclusion of mobility of the amino acid side chains that interact with the crystallized ligand (Table 1).

4.1.4. Virtual Screening. The search of new candidates was performed over Drugbank ${ }^{41}$ and ZINC-FDA databases. ${ }^{42}$ Drugbank includes 7818 compounds of FDA-approved compounds, nutraceuticals, and drugs in the experimental stage. The structures were downloaded from the database in SDF format, and the $2 \mathrm{D}$ coordinates were converted to $3 \mathrm{D}$ with OpenBabel2.1.2 software. ${ }^{64}$ Compounds with molecular weight lower than 160 or higher than 600 were discarded. The remaining 5775 molecules were protonated at physiological $\mathrm{pH}$ (7.4) and submitted to energy minimization with OpenBabel2.1.2:750 steps of Monte Carlo search and geometry optimization with MMFF94 force field followed by 300 cycles of conjugated gradient method for the energy minimization for the best conformation. ${ }^{64}$ Regarding the ZINC database, we employed a subset of compounds that contains 3358 structures of FDA-approved drugs (with their tautomers and other isomers $)^{42}$ in a ready to dock format (pdbqt files).

4.2. Biological Assays. 4.2.1. Electrophysiology. Patchclamp experiments were performed in HEK293 cell lines stably expressing $\mathrm{hNa}_{\mathrm{V}} 1.2$ (a kind gift of GlaxoSmithKline, Stevenage, UK) that have been described in the literature. ${ }^{65,66}$

4.2.1.1. Cell Culture. HEK293 cell lines were cultured in DMEM F12, containing 10\% fetal bovine serum (FBS) and $0.5 \%$ Geneticin G418 sulfate. Cells were grown in a $95 \% \mathrm{O}_{2} /$ $5 \% \mathrm{CO}_{2}$ atmosphere at $37{ }^{\circ} \mathrm{C}$ with $95 \%$ humidity. Cells were passaged using trypsin when they reached $70 \%$ confluence. On the day of the experiment, cells were detached using Accutase (ThermoFisher). An appropriate volume of cells suspension was added to a conical tube and centrifuged at $1000 \mathrm{rpm}$ for 5 min. The pellet was resuspended in $5 \mathrm{~mL}$ of DMEM F12 medium without FBS and Geneticin and used for patch-clamp experiments. Immediately before electrophysiological recordings, cells were allowed to settle onto the coverglass bottom of a $3 \mathrm{~mL}$ experimental chamber. Data were collected within 4-6 $\mathrm{h}$ after cell isolation.

4.2.1.2. Whole-Cell Voltage-Clamp Recordings. Cells were observed with a mechanically stabilized inverted microscope (Telaval 3, Carl Zeiss, Jena, Germany) equipped with a $40 \times$ objective lens. The test solutions were applied through a multibarreled pipet positioned close to the target cell. After each experiment on a single cell, the experimental chamber was replaced by another one containing a new sample of cells. All experiments were performed at room temperature $\left(\sim 22{ }^{\circ} \mathrm{C}\right)$.

The standard tight-seal whole-cell configurations of the patch-clamp technique ${ }^{67,68}$ were used to record macroscopic currents. Glass pipettes were drawn from WPI PG52165-4 glass on a two-stage vertical micropipette puller (PP-83, Narishige Scientific Instrument Laboratories, Tokyo, Japan), and pipet resistance ranged from 1.5 to $2.5 \mathrm{M} \Omega$.

Whole-cell currents were filtered with a 4-pole lowpass Bessel filter (Axopatch 200A amplifier) at $2 \mathrm{kHz}$ and digitized (Digidata 1440, Molecular devices) at a sampling frequency of $200 \mathrm{kHz}(5 \mu \mathrm{s})$. The experimental recordings were stored on a computer hard disk for later analysis. Cells were placed in a 
recording chamber and perfused with extracellular solution containing (in $\mathrm{mM}$ ): $\mathrm{NaCl}, 140 ; \mathrm{KCl}, 5 ; \mathrm{CaCl}_{2}, 2 ; \mathrm{MgCl}_{2}, 1$; HEPES, 10; glucose, 11; $\mathrm{pH}$ was adjusted to 7.4 with $\mathrm{NaOH}$. In the cases where current was greater than $1 \mathrm{nA}, \mathrm{NaCl}$ was reduced to $50 \mathrm{mM}$ in extracellular solution (by replacing it with $\mathrm{N}$-methyl-D-glucamine) to reduce the effect of series resistance in Nav currents. The patch electrodes were filled with pipet solution containing (in $\mathrm{mM}$ ): CsF, 140; EGTA, 10; HEPES, 10; $\mathrm{NaCl}, 5 ; \mathrm{MgCl}_{2}, 2$; the $\mathrm{pH}$ was adjusted to 7.3 with $\mathrm{CsOH}$. Whole-cell current stability was monitored by applying successive $10 \mathrm{~ms}$ voltage steps (from a holding potential of $-70 \mathrm{mV}$ to a test potential of $-20 \mathrm{mV}$ ), discarding cells in which the peak current amplitude did not remain constant in time. After the current was stable $(\sim 10 \mathrm{~min})$, the same voltage-clamp step protocol was applied in the control or in the presence of compound candidates. We also included the inhibitory effect of $100 \mu \mathrm{M}$ Phenytoin for comparative purposes. In all cases, the inhibition was observed as the decay in the peak amplitude.

4.2.1.3. Statistical Analyses. The effects of the compounds on Nav current were compared using a paired $t$ test. A $p$ value $<0.05$ was considered statistically significant. All data were expressed as mean \pm SEM.

4.2.1.4. Drugs and Reagents. CsF, EGTA, and $\mathrm{Na}_{2} \mathrm{ATP}$ were purchased from Sigma Chemical Co. Accustase was purchased from ThermoFisher. All other reagents including DMEM medium and FBS were of analytical grade and purchased from local suppliers. $N, N^{\prime}$-Diphenethylsulfamide, Losartan, and Valsartan were dissolved in dimethyl sulfoxide (DMSO). Fresh aliquots of stock solutions of compound in DMSO were added to the bath solution on the day of the experiment. Appropriate amounts of DMSO were added to all control solutions without compound.

4.2.2. In Vivo Assays. The evaluation of the anticonvulsant activity followed the Anticonvulsant Drug Development (ADD) Program of the National Institute of Health. ${ }^{4}$ Adult male albino mice $(18-23 \mathrm{~g})$ of the same age and weight have been selected, in order to minimize biological variability. The animals were provided by the Faculty of Veterinary, National University of La Plata. They were maintained under a regime of a $12 \mathrm{~h} \mathrm{light/dark} \mathrm{cycle} \mathrm{and} \mathrm{allowed} \mathrm{free} \mathrm{access} \mathrm{to} \mathrm{food} \mathrm{and}$ water, except during the time they were removed from their cages for testing. Every effort was made to minimize animal stress. The animal care for the experimental protocols was conducted in accordance with the NIH guidelines for the Care and Use of Laboratory Animals, and it was approved by the Ethical Committee of Exact Sciences Faculty of University of La Plata.

The candidates were administered intraperitoneally in physiological solution (volume of $0.01 \mathrm{~mL} / \mathrm{g}$ body weight). We selected the concentrations of 30 and $100 \mathrm{mg} / \mathrm{kg}$ for the candidates, and the assays were performed at 0.5 and $4 \mathrm{~h}$.

Maximal electroshock seizures were provoked in mice by delivering a $60 \mathrm{~Hz} / 50 \mathrm{~mA}$ electrical stimulus for $0.2 \mathrm{~s}$ via ear clip electrodes by means of UGO Basile equipment. In these conditions, normal mice experience maximal seizures, characterized by a short period of tonic flexion followed by a longer period of tonic extension of the hind limbs and a final clonic episode. ${ }^{4}$ The anticonvulsant activity of the candidates was detected through the absence of the hind limbs extension.

\section{ASSOCIATED CONTENT}

\section{Supporting Information}

The Supporting Information is available free of charge on the ACS Publications website at DOI: 10.1021/acs.jcim.7b00721.

Table S1, value of the scores achieved for each domain of the closed and open models of Nav1.2; Figure S1, Ramachandran plots of the overall architecture of the final Nav1.2 closed model; Figure S2, results of QMEANbrane software for the Nav1.2 closed model; Figure S3, comparison between open and closed experimental conformations of the $\mathrm{NaK}$ pore (PDB entries: 3E86 and 2AHY) and between this open conformation with the NavAb template (PDB entry: RVY); Figure S4, Ramachandran plots of the overall architecture of the final Nav1.2 open model; Figure S5, results of QMEANbrane software for Nav1.2 open model (PDF)

\section{AUTHOR INFORMATION}

\section{Corresponding Author}

*E-mail: lgavernet@biol.unlp.edu.ar. Tel.: +542214235333 int 41.

\section{ORCID}

Luciana Gavernet: 0000-0001-9285-3788

\section{Notes}

The authors declare no competing financial interest.

\section{ACKNOWLEDGMENTS}

L.E.B.B. is a member of the Facultad de Ciencias Exactas, Universidad Nacional de La Plata. L.G., P.M., and V.M. are members of Consejo Nacional de Investigaciones Cientificas y Técnicas de la República Argentina (CONICET). P.H.P., L.L.S., M.L.V., and N.E. are fellowship holders of CONICET. S.G. is a fellowship holder of La Plata University. This research was supported in part by the Agencia de Promoción Cientifica y Tecnológica (PICT 3175/2013), CONICET, and Universidad Nacional de La Plata, Argentina.

\section{REFERENCES}

(1) W.H.O. Fact Sheet No. 999: Epilepsy; WHO: Geneva, 2015

(2) Löscher, W.; Klitgaard, H.; Twyman, R. E.; Schmidt, D. New Avenues for Anti-Epileptic Drug Discovery and Development. Nat. Rev. Drug Discovery 2013, 12, 757-776.

(3) Meldrum, B. S.; Rogawski, M. A. Molecular Targets for Antiepileptic Drug Development. Neurotherapeutics 2007, 4, 18-61.

(4) Stables, J. P.; Kupferberg, H. J. The NIH Anticonvulsant Drug Development (ADD) Program: Preclinical Anticonvulsant Screening Project. In Molecular and cellular targets for antiepileptic drugs; Avanzani, G., Regesta, G., Tanganelli, P., Avoli, M., Eds.; John Libbey Eurotext: Paris, 1997; pp 191-198.

(5) Rogawski, M. A.; Löscher, W. The neurobiology of antiepileptic drugs. Nat. Rev. Neurosci. 2004, 5, 553-564.

(6) Kambli, L.; Bhatt, L. K.; Oza, M.; Prabhavalkar, K. Novel therapeutic targets for epilepsy intervention. Seizure 2017, 51, 27-34.

(7) Younus, I.; Reddy, D. S. A resurging boom in new drugs for epilepsy and brain disorders. Expert Rev. Clin. Pharmacol. 2018, 11, $27-45$.

(8) Wood, M. D.; Gillard, M. Evidence for a differential interaction of brivaracetam and levetiracetam with the synaptic vesicle $2 \mathrm{~A}$ protein. Epilepsia 2017, 58, 255-262.

(9) Vohora, D.; Saraogi, P.; Yazdani, M. a.; Bhowmik, M.; Khanam, R.; Pillai, K. K. Recent Advances in Adjunctive Therapy for Epilepsy: 
Focus on Sodium Channel Blockers as Third-Generation Antiepileptic Drugs. Drugs Today 2010, 46, 265.

(10) Brodie, M. J. Antiepileptic Drug Therapy the Story so Far. Seizure 2010, 19, 650-655.

(11) Fantini, M.; Rivara, M.; Zuliani, V.; Kalmar, C. L.; Vacondio, F.; Silva, C.; Baheti, A. R.; Singh, N.; Merrick, E. C.; Katari, R. S.; Cocconcelli, G.; Ghiron, C.; Patel, M. K. 2,4(5)-Diarylimidazoles as Inhibitors of hNaV1.2 Sodium Channels: Pharmacological Evaluation and Structure-Property Relationships. Bioorg. Med. Chem. 2009, 17, $3642-3648$.

(12) Yu, F. H.; Yarov-Yarovoy, V.; Gutman, G. A.; Catterall, W. A. Overview of molecular relationships in the voltage-gated ion channel superfamily. Pharm. Rev. 2005, 57, 387-395.

(13) Catterall, W. A.; Goldin, A. L.; Waxman, S. G. International Union of Pharmacology. XLVII. Nomenclature and structure-function relationships of voltage-gated sodium channels. Pharmacol. Rev. 2005, 57, 397-409.

(14) Nardi, A.; Damann, N.; Hertrampf, T.; Kless, A. Advances in Targeting Voltage-Gated Sodium Channels with Small Molecules. ChemMedChem 2012, 7, 1712-1740.

(15) Bagal, S. K.; Marron, B. E.; Owen, R. M.; Storer, R. I.; Swain, N. A. Voltage Gated Sodium Channels as Drug Discovery Targets. Channels 2015, 9, 360-366.

(16) Tang, F.; Hartz, A. M. S.; Bauer, B. Drug-Resistant Epilepsy: Multiple Hypotheses, Few Answers. Front. Neurol. 2017, 8, 301.

(17) French, J. A. P-Glycoprotein Expression and Antiepileptic Drug Resistance. Lancet Neurol. 2013, 12, 732-733.

(18) Oprea, T. I.; Overington, J. P. Computational and Practical Aspects of Drug Repositioning. Assay Drug Dev Technol. 2015, 1, 28 35 .

(19) Ma, D. L.; Chan, D. S.-H.; Leung, C.-H. Drug Repositioning by Structure-Based Virtual Screening. Chem. Soc. Rev. 2013, 42, 21302141.

(20) Gavernet, L.; Elvira, J. E.; Samaja, G. a; Pastore, V.; Cravero, M. S.; Enrique, A.; Estiu, G.; Bruno-Blanch, L. E. Synthesis and Anticonvulsant Activity of Amino Acid-Derived Sulfamides. J. Med. Chem. 2009, 52, 1592-15601.

(21) Gavernet, L.; Barrios, I. A.; Cravero, M. S.; Bruno-Blanch, L. E. Design, Synthesis, and Anticonvulsant Activity of Some Sulfamides. Bioorg. Med. Chem. 2007, 15, 5604-5014.

(22) Villalba, M. L.; Enrique, A. V.; Higgs, J.; Castaño, R. A.; Goicoechea, S.; Taborda, F. D.; Gavernet, L.; Lick, I. D.; Marder, M.; Bruno Blanch, L. E. Novel Sulfamides and Sulfamates Derived from Amino Esters: Synthetic Studies and Anticonvulsant Activity. Eur. J. Pharmacol. 2016, 774, 55-63.

(23) Levy, R. H.; Mattson, R. H.; Meldrum, B. S.; Perucca, E. Antiepileptic Drugs, Fifth ed.; Lippincott Williams \& Wilkins: Philadelphia, 2002; p 44.

(24) Ragsdale, D. S.; McPhee, J. C.; Scheuer, T.; Catterall, W. A. Common molecular determinants of local anesthetic, antiarrhythmic, and anticonvulsant block of voltage-gated $\mathrm{Na}+$ channels. Proc. Natl. Acad. Sci. U. S. A. 1996, 93, 9270-9275.

(25) Zhang, J.; Yang, J.; Jang, R; Zhang, Y. GPCR-I-TASSER: A hybrid approach to $G$ protein-coupled receptor structure modeling and the application to the human genome. Structure 2015, 23, 15381549.

(26) Berman, H. M.; Westbrook, J.; Feng, Z.; Gilliland, G.; Bhat, T. N.; Weissig, H.; Shindyalov, I. N.; Bourne, P. E. The Protein Data Bank. Nucleic Acids Res. 2000, 28, 235-242.

(27) Payandeh, J.; Scheuer, T.; Zheng, N.; Catterall, W. A. The crystal structure of a voltage-gated sodium channel. Nature 2011, 475, $353-358$.

(28) Case, D.; Darden, T. A.; Cheatham, T. E.; Simmerling, C. L.; Wang, J.; Duke, R. E.; Duke, R. E.; Luo, R.; Crowley, M.; Walker, R.; Zhang, W.; Merz, K. M.; Wang, B.; Hayik, S.; Roitberg, A.; Seabra, G.; Kolossvary, I.; Wong, K. F.; Paesani, F.; Vanicek, J.; Wu, X.; Brozell, S. R.; Steinbrecher, T.; Gohlke, H.; Yang, L.; Tan, C.; Mongan, J.; Hornak, V.; Cui, G.; Mathews, D. H.; Seetin, M. G.; Sagui, C.; Babin,
V.; Kollman, P. A. AMBER 11 Users' Manual; University of California: San Francisco, 2010; pp 1-304.

(29) Naylor, C. E.; Bagnéris, C.; DeCaen, P. G.; Sula, A.; Scaglione, A.; Clapham, D. E.; Wallace, B. Molecular Basis of Ion Permeability in a Voltage-gated Sodium Channel. EMBO J. 2016, 35, 820-830.

(30) Studer, G.; Biasini, M.; Schwede, T. Assessing the local structural quality of transmembrane protein models using statistical potentials (QMEANBrane). Bioinformatics 2014, 30, i505-i511.

(31) Tomašić, T.; Hartzoulakis, B.; Zidar, N.; Chan, F.; Kirby, R. W.; Madge, D. J.; Peigneur, S.; Tytgat, J.; Kikelj, D. Ligand- and Structure-Based Virtual Screening for Clathrodin-Derived Human Voltage-Gated Sodium Channel Modulators. J. Chem. Inf. Model. 2013, 53, 3223-3232.

(32) Alam, A.; Jiang, Y. High-Resolution Structure of the Open NaK Channel. Nat. Struct. Mol. Biol. 2009, 16, 30-34.

(33) Webb, B.; Sali, A. Comparative Protein Structure Modeling Using MODELLER. In Current Protocols in Bioinformatics; John Wiley \& Sons, Inc.: Hoboken, NJ, USA, 2014; p 5.6.1-5.6.37.

(34) Martí-Renom, M.; Stuart, A.; et al. Comparative Protein Structure Modeling of Genes and Genomes. Annu. Rev. Biophys. Biomol. Struct. 2000, 29, 291-325.

(35) Fiser, A.; Do, K. D.; Sali, A. Modeling of loops in protein structures. Protein Sci. 2000, 9, 1753-1773.

(36) Sali, A.; Blundell, T. L. Comparative Protein Modelling by Satisfaction of Spatial Restraints. J. Mol. Biol. 1993, 234, 779-815.

(37) Bento, P.; Gaulton, A.; Hersey, A.; Bellis, L. J.; Chambers, J.; Davies, M.; Kruger, F. A.; Light, Y.; Mak, L.; McGlinchey, S.; Nowotka, M.; Papadatos, G.; Santos, R.; Overington, J. P. The ChEMBL bioactivity database: An update. Nucleic Acids Res. 2014, 42, D1083-D1090.

(38) Trott, O.; Olson, A. J. AutoDock Vina: improving the speed and accuracy of docking with a new scoring function, efficient optimization, and multithreading. J. Comput. Chem. 2010, 31, 455461.

(39) Boiteux, C.; Vorobyov, I.; French, R. J.; French, C.; YarovYarovoy, V.; Allen, T. W. Local Anesthetic and Antiepileptic Drug Access and Binding to a Bacterial Voltage-Gated Sodium Channel. Proc. Natl. Acad. Sci. U. S. A. 2014, 111 (36), 13057-13062.

(40) Rizzi, A.; Fioni, A. Virtual screening using PLS discriminant analysis and ROC curve approach: an application study on PDE4 inhibitors. J. Chem. Inf. Model. 2008, 48, 1686-1692.

(41) Law, V.; Knox, C.; Djoumbou, Y.; Jewison, T.; Guo, A. C.; Liu, Y.; Maciejewski, A.; Arndt, D.; Wilson, M.; Neveu, V.; Tang, A.; Gabriel, G.; Ly, C.; Adamjee, S.; Dame, Z. T.; Han, B.; Zhou, Y.; Wishart, D. S. DrugBank 4.0: shedding new light on drug metabolism. Nucleic Acids Res. 2014, 42, D1091-D1097.

(42) Irwin, J. J.; Sterling, T.; Mysinger, M. M.; Bolstad, E. S.; Coleman, R. G. ZINC: a free tool to discover chemistry for biology. J. Chem. Inf. Model. 2012, 52, 1757-1768.

(43) Palestro, P. H.; Gavernet, L.; Estiu, G. L.; Bruno Blanch, L. E. Docking Applied to the Prediction of the Affinity of Compounds to PGlycoprotein. BioMed Res. Int. 2014, 2014, 358425.

(44) Szewczyk, P.; Tao, H.; McGrath, A. P.; Villaluz, M.; Rees, S. D.; Lee, S. C.; Doshi, R.; Urbatsch, I. L.; Zhang, Q.; Chang, G. Snapshots of Ligand Entry, Malleable Binding and Induced Helical Movement in P-Glycoprotein. Acta Crystallogr., Sect. D: Biol. Crystallogr. 2015, 71, $732-741$

(45) Aller, S. G.; Yu, J.; Ward, A.; Weng, Y.; Chittaboina, S.; Zhuo, R.; Harrel, P. M.; Trinh, Y. T.; Zhang, Q.; Urbatsch, I. L.; Chang, G. Structure of P-glycoprotein reveals a molecular basis for poly-specific drug binding. Science 2009, 323, 1718-1722.

(46) Martin, C.; Berridge, G.; Higgins, C. F.; Mistry, P.; Charlton, P.; Callaghan, R. Communication between multiple drug binding sites on P-glycoprotein. Mol. Pharmacol. 2000, 58, 624-632.

(47) Broccatelli, F.; Carosati, E.; Neri, A.; Frosini, M.; Goracci, L.; Oprea, T. I.; Cruciani, G. A Novel Approach for Predicting PGlycoprotein (ABCB1) Inhibition Using Molecular Interaction Fields. J. Med. Chem. 2011, 54, 1740-1751. 
(48) Liu, B. G.; Zhuang, X. L.; Li, S. T.; Xu, G. H. The effects of ropivacaine on sodium currents in dorsal horn neurons of neonatal rats. Anesth. Analg. 2000, 90, 1034-1038.

(49) Cheng, H. W.; Yang, H. T.; Zhou, J. J.; Ji; Zhu, H. Y. Pharmacological modulation of brain Nav1.2 and cardiac Nav1.5 subtypes by the local anesthetic ropivacaine. Neurosci. Bull. 2010, 26, 289-296.

(50) Niwa, R.; Honjo, H.; Kodama, I.; Maruyama, K.; Toyama, J. Na + channel blocking efects of cibenzoline on guinea-pig ventricular cells. Eur. J. Pharmacol. 1998, 352, 317-327.

(51) Uebachs, M.; Albus, C.; Opitz, T.; Isom, L.; Niespodziany, I.; Wolff, C.; Beck, $\mathrm{H}$. Loss of $\beta 1$ accessory $\mathrm{Na}+$ channel subunits causes failure of carbamazepine, but not of lacosamide, in blocking highfrequency firing via differential effects on persistent $\mathrm{Na}+$ currents. Epilepsia 2012, 53, 1959-1967.

(52) Keppel Hesselink, J. M.; Kopsky, D. J. Phenytoin: 80 years young, from epilepsy to breast cancer, a remarkable molecule with multiple modes of action. J. Neurol. 2017, 264, 1617-1621.

(53) LaPenna, P.; Tormoehlen, L. M. The Pharmacology and Toxicology of Third-Generation Anticonvulsant Drugs. J. Med. Toxicol. 2017, 13, 329-342.

(54) Yuen, E. S. M.; Trocóniz, I. F. Can pentylenetetrazole and maximal electroshock rodent seizure models quantitatively predict antiepileptic efficacy in humans? Seizure 2015, 24, 21-27.

(55) The UniProt Consortium. UniProt: the universal protein knowledgebase. Nucleic Acids Res. 2017, 45, D158-D169.

(56) Bento, P.; Gaulton, A.; Hersey, A.; Bellis, L. J.; Chambers, J.; Davies, M.; Kruger, F. A.; Light, Y.; Mak, L.; McGlinchey, S.; Nowotka, M.; Papadatos, G.; Santos, R.; Overington, J. P. The ChEMBL bioactivity database: An update. Nucleic Acids Res. 2014, 42, D1083-D1090.

(57) Lenkey, N.; Karoly, R.; Lukacs, P.; Vizi, E. S.; Sunesen, M.; Fodor, L.; Mike, A. Classification of drugs based on properties of sodium channel inhibition: a comparative automated patch-clamp study. PLoS One 2010, 5, e15568.

(58) Schwab, D.; Fischer, H.; Tabatabaei, A.; Poli, S.; Huwyler, J. Comparison of in vitro P-glycoprotein screening assays: recommendations for their use in drug discovery. J. Med. Chem. 2003, 46, 17161725.

(59) Polli, J. W.; Wring, S. A.; Humphreys, J. E.; Huang, L.; Morgan, J. B.; Webster, L. O.; Serabjit-Singh, C. S. Rational use of in vitro Pglycoprotein assays in drug discovery. J. Pharmacol. Exp. Ther. 2001, 299, 620-628.

(60) Feng, B.; Mills, J. B.; Davidson, R. E.; Mireles, R. J.; Janiszewski, J. S.; Troutman, M. D.; de Morais, S. M. In vitro P-glycoprotein assays to predict the in vivo interactions of P-glycoprotein with drugs in the central nervous system. Drug Metab. Dispos. 2008, 36, 268-275.

(61) Mahar Doan, K. M.; Humphreys, J. E.; Webster, L. O.; Wring, S. A.; Shampine, L. J.; Serabjit-Singh, C. J.; Adkison, K. K.; Polli, J. W. Passive permeability and P-glycoprotein-mediated efflux differentiate central nervous system (CNS) and non-CNS marketed drugs. J. Pharmacol. Exp. Ther. 2002, 303, 1029-1037.

(62) Di Ianni, M. E.; Enrique, A. V.; Palestro, P. H.; Gavernet, L.; Talevi, A.; Bruno-Blanch, L. E. Several New Diverse Anticonvulsant Agents Discovered in a Virtual Screening Campaign Aimed at Novel Antiepileptic Drugs to Treat Refractory Epilepsy. J. Chem. Inf. Model. 2012, 52, 3325-3330.

(63) Couyoupetrou, M.; Gantner, M. E.; Di Ianni, M. E.; Palestro, P. H.; Enrique, A. V.; Gavernet, L.; Ruiz, M.e.; Pesce, G.; Bruno-Blanch, L. E.; Talevi, A. Computer-Aided Recognition of ABC Transporters Substrates and Its Application to the Development of New Drugs for Refractory Epilepsy. Mini-Rev. Med. Chem. 2017, 17, 205-215.

(64) O’Boyle, N. M.; Banck, M.; James, C. A.; Morley, C.; Vandermeersch, T.; Hutchison, G. R. Open Babel: An Open chemical toolbox. J. Cheminf. 2011, 3, 33.

(65) Chen, Y. H.; Dale, T. J.; Romanos, M. A.; Whitaker, W. R.; Xie, X. M.; Clare, J. J. Cloning, Distribution and Functional Analysis of the Type III Sodium Channel from Human Brain. Eur. J. Neurosci. 2000, 12, 4281-4289.
(66) Burbidge, S. a; Dale, T. J.; Powell, A. J.; Whitaker, W. R. J.; Xie, X. M.; Romanos, M. a; Clare, J. J. Molecular Cloning, Distribution and Functional Analysis of the NA(V)1.6. Voltage-Gated Sodium Channel from Human Brain. Mol. Brain Res. 2002, 103, 80-90.

(67) Mantegazza, M.; Yu, F. H.; Powell, A. J.; Clare, J. J.; Catterall, W. A.; Scheuer, T. Molecular Determinants for Modulation of Persistent Sodium Current by G-Protein Subunits. J. Neurosci. 2005, $25,3341-3349$.

(68) Hamill, O.; Marty, A.; Neher, E.; Sakmann, B.; Sigworth, F. Improved Patch-Clamp Techniques for High-Resolution Current Recording from Cells and Cell-Free Membrane Patches. Pfluegers Arch. 1981, 391, 85-100. 